\title{
Spinal Brucellosis: A Very Subtle Cause
}

\section{Soumil Singhal ${ }^{1}$}

${ }^{1}$ Fellow in intervention radiology, Department of intervention radiology and intervention oncology, BGS Gleneagles Global Hospital Bangalore.

\section{Abstract}

Osteoarticular brucellosis is a serious complication of human brucellosis. Brucellosis of the spine is not a very common cause of back pain in an Indian population. I present here a case presenting with an unusual backache diagnosed to finally have brucellosis and its clinical presentation and imaging findings.

Keywords: Brucellosis, spondylitis, abscess, imaging.

Corresponding Author: Dr. Soumil Singhal, Fellow in intervention radiology, Department of intervention radiology and intervention oncology, BGS Gleneagles Global Hospital Bangalore

Received: August 2018

Accepted: September 2018

\section{Introduction}

Brucellosis is a zoonotic infection which is transmitted mainly by direct contact with infected animal, inhalation of the aerosol or by ingestion of infected products. Osteoarticular brucellosis is the most important complication. Brucellosis of the spine is not a very common cause of back pain in an Indian population. I present here a case presenting with unusual backache diagnosed to finally have brucellosis.

\section{Case History}

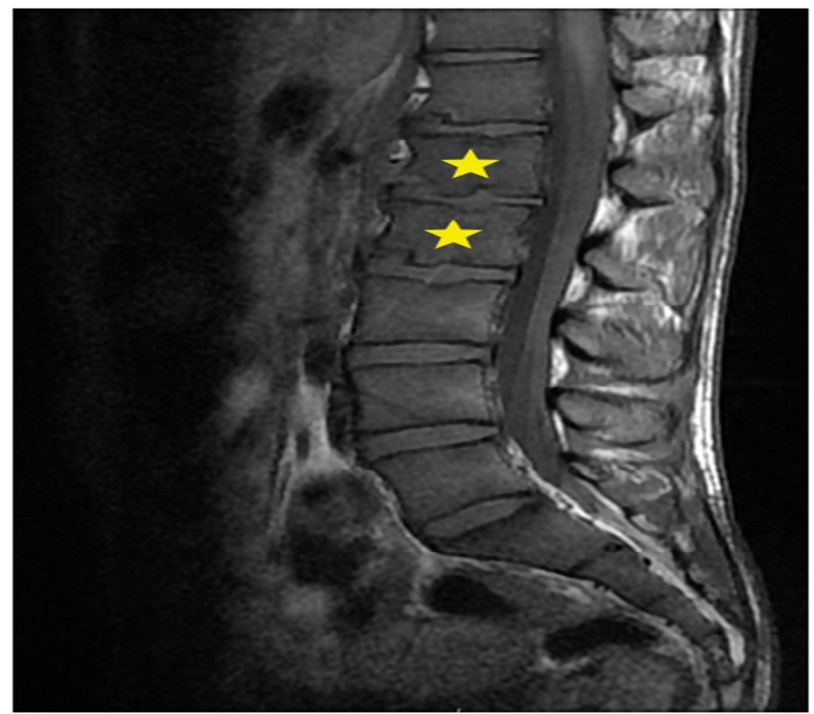

Figure 1: Saggital T1- Weighted Image shows end plate changes in $\mathrm{L} 1, \mathrm{~L} 2$ vertebral bodies (Yellow star)

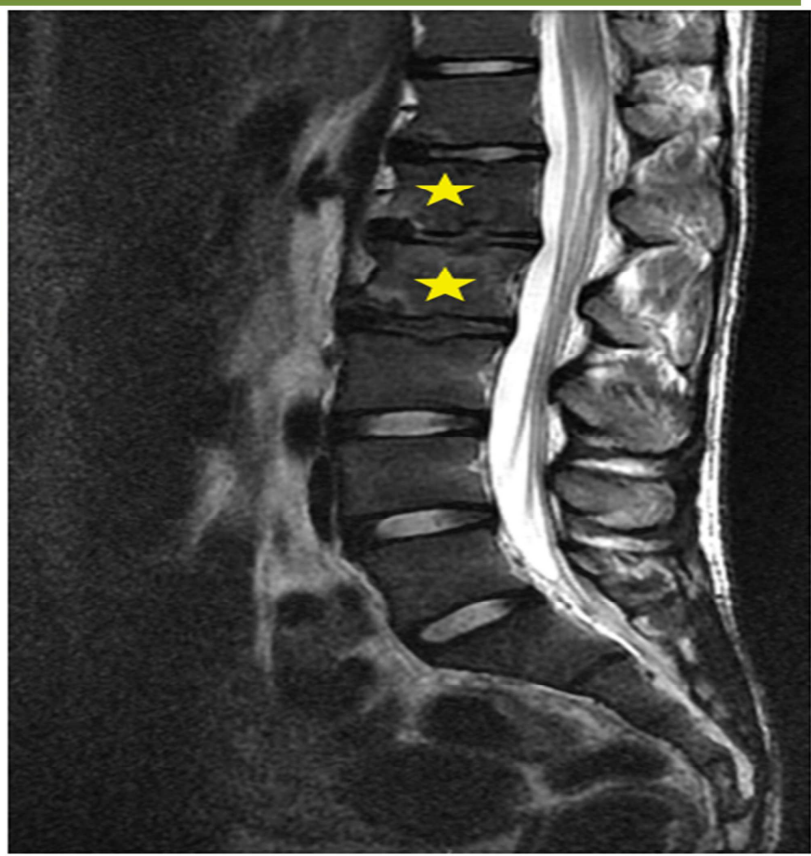

Saggital T2- Weighted Image shows hyperintense signal at the $L 1$ and $L 2$ vertebral bodies (Yellow star)

A 27-year-old male patient working in the middle east presented to the hospital with complaints of low back pain which was gradual in onset and diffuse in nature. It was associated with on and off fever. the pain aggravated by slight movement and relieved on medication. On clinical examination mild tenderness was noted in right sacroiliac joint, SLR was negative, no focal neurological deficit were noted. Patients blood evaluation showed Mild anemia (11 $\mathrm{mg} / \mathrm{dl}$ ), Raised ESR (50), Raised CRP levels (39.96), Elevated liver enzymes (AST- 52, ALT-66, ALP - 131). 
Xray of the spine did not show any significant findings following which MRI was performed. MRI Lumbar spine showed endplate changes in the L1 and L2 vertebral bodies [Figure 1 \& 2] with surrounding areas showing STIR hyperintensity [Figure 3]. On Post-contrast scan, corresponding areas show enhancement. There is a patch area of enhancement seen in the paravertebral region [Figure $4 \& 5$ ]. A initial diagnosis of infective discitis was made. Patients blood was evaluated further and it was IgM Brucella antibody positive and culture grew Brucella melitensis. Patient was stated on antibiotic treatment. On Follow up patient showed improvement.

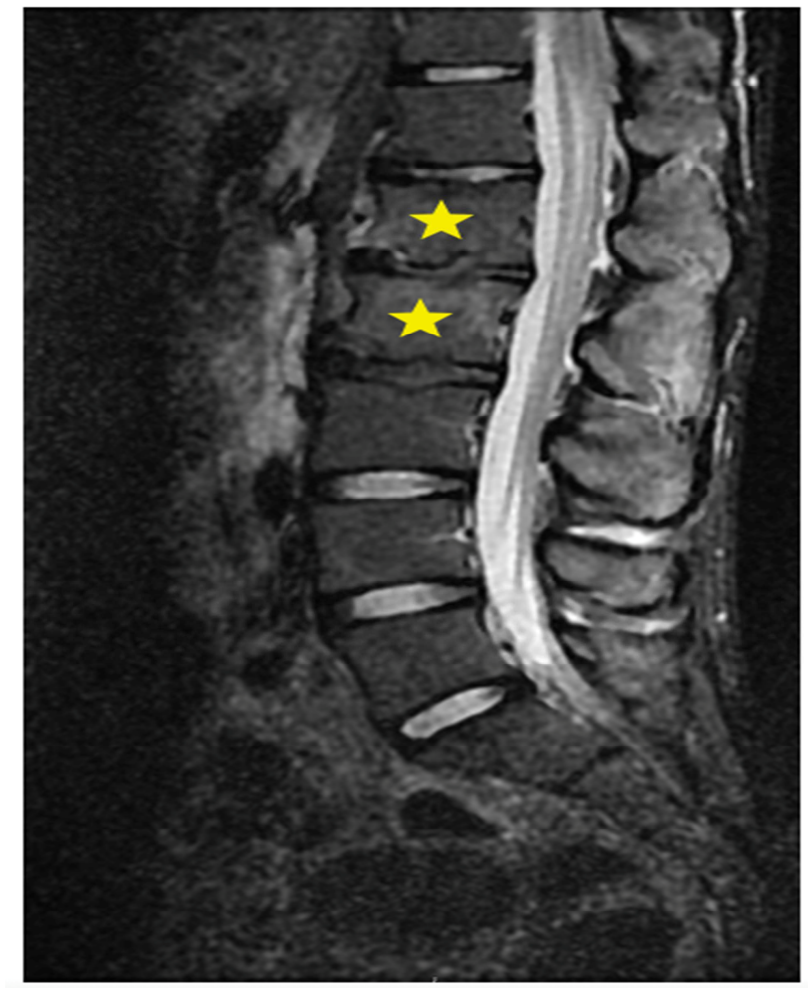

STIR hyperintense signal at L1 and L2 levels (Yellow star)

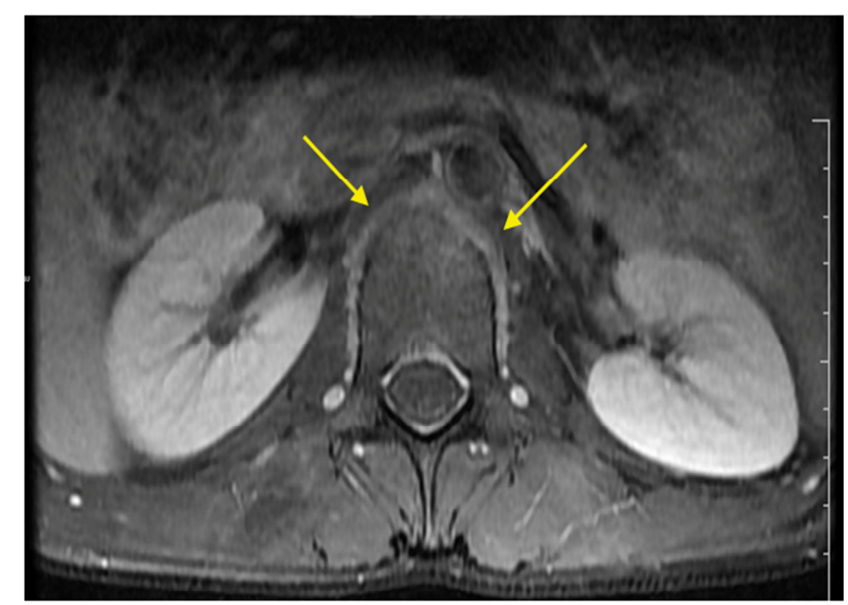

Axial Post contrast enhancement seen in the corresponding area with a patchy para vertebral component (Arrow)
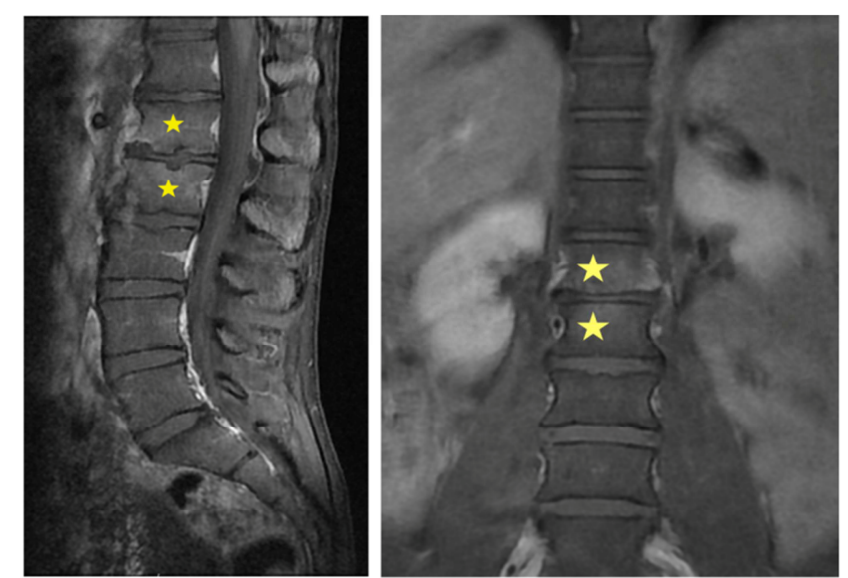

Saggital Post contrast Images show enhancement in the L1, L2 vertebral body end plates (Yellow star)

\section{Discussion}

Brucellosis is a zoonotic infection which is transmitted mainly by direct contact with infected animal, inhalation of the aerosol or by ingestion of infected products. Human Brucellosis remains a public health problem in most developing conditions and is mainly caused by Brucella abortus, Brucella Suis, Brucella Canis and Brucella Melitensis. Reticuloendothelial rich organs are mainly targeted and most frequent organ targeted is the musculoskeletal system. ${ }^{[1,2]}$ Spine and large joints are frequently affected. ${ }^{[2]}$ The incidence of spinal involvement can range from $9-58 \%$. ${ }^{[3,4]}$

The lumbar spine is most commonly involved particularly L4-5, L5-S1. ${ }^{[4]}$

Clinical Perspective: Patient presents with non-specific complaints such as malaise, backache, fever, and anorexia. The patient may develop neurological symptoms such as paraesthesia and weakness due to spinal cord compression which when not treated can further progress to neurobrucellosis. ${ }^{[5]}$ Diagnosis of the condition can be difficult, however proper history, clinical, laboratory and radiological investigation can help diagnose the condition. Imaging Perspective: Spinal involvement can either be a) Focal or b) Diffuse. Focal involvement presents as erosions in the anterosuperior endplate and diffuse involvement presents as the erosion of entire endplate or vertebral bodies and spreads to the adjacent spine by subligamentous or vascular route. Posterior elements are rarely involved. Epidural involvement is also seen frequently. Radiography is non-specific and not helpful in the diagnosis of an acute condition. Bone scintigraphy is highly sensitive and shows increased uptake [1]. CT shows either a focal area of erosion, sclerosis, osteophytes and disc air or diffuse destruction with epidural extension. MRI is the modality of choice, it is more sensitive in locating the level of involvement, epidural and paraspinal involvement. T2 weighted images are especially sensitive as it demonstrates hyperintense signals. Postcontrast scan shows enhancement of the inflammatory component. 


\section{Conclusion}

1. Spinal brucellosis can easily be overlooked, however since its a destructive condition, it needs an early and correct diagnosis with the initiation of treatment.

2. MRI is the imaging modality of choice. It is highly sensitive and can help differentiate from various other infectious causes of spondylodiscitis.

\section{References}

1. Al-Shahed MS, Sharif HS, Haddad MC, Aabed MY, Sammak BM, Mutairi MA (1994) Imaging features of musculoskeletal brucellosis..
Radiographics Mar;14(2):333-48

2. Lifeso RM, Harder E, McCorkell SJ. (1985) Spinal brucellosis. J Bone joint Surg [Br] 1985; 67: 345-351.

3. Aygen, B., Doganay, M., Sumerkan, B., Yildiz, O., and Kayabas, U (2002) Clinical manifestations, complications and treatment of brucellosis: a retrospective evaluation of 480 patients. Med Mal Infect. 2002; 32: 485493

4. Colmenero JD, Reguera JM, Fernández-Nebro A, Cabrera-Franquelo F. (1991) Osteoarticular complications of brucellosis. Ann Rheum Dis. 1991; 50: $23-26$

5. Doganay, M. and Aygen, B (2003) Human brucellosis: an overview. Int J Infect Dis. 2003; 7: 173-182

Copyright: ( ) the author(s), publisher. Asian Journal of Medical Radiological Research is an Official Publication of "Society for Health Care \& Research Development". It is an open-access article distributed under the terms of the Creative Commons Attribution Non-Commercial License, which permits unrestricted non-commercial use, distribution, and reproduction in any medium, provided the original work is properly cited.

How to cite this article: Singhal S. Spinal Brucellosis: A Very Subtle Cause. Asian J. Med. Radiol. Res. 2018;6(1):9-11.

DOI: dx.doi.org/10.21276/ajmrr.2018.6.1.3

Source of Support: Nil, Conflict of Interest: None declared. 